\title{
Wavelet neural network for vector prediction to fill-in missing image blocks in wireless transmission
}

\begin{abstract}
In this paper, the problem of information loss in block-coded images in wireless transmissions over a packet network is addressed. The proposed method achieves accurate values by minimizing the mean square error (MSE) between the border coefficients of the lost block to recovering the missing coefficients. The edge direction of the lost block is found using the best match from two boundaries of neighboring blocks. Further, a multilayer perceptron (MLP) neural network architecture is designed using a supervised training procedure. By implementing a nonlinear vector predictor, the architecture is used to predict the blocks that contain edges. Experimental results show that the performance efficiency of the MLP can be evaluated in terms of the visual quality and the MSE of the predicted image. The proposed methods provide significant improvements in terms of loss concealment and artifacts, especially those associated with edges.
\end{abstract}

Keyword: Block-coded images; Lost block; Neural network; Vector predictor 